\title{
Subarachnoid hemorrhage due to ruptured intracranial aneurysm following posterior reversible encephalopathy syndrome
}

\author{
Takamasa Nanba, Hiroshi Kashimura, Hiroaki Saura, Masaru Takeda \\ Department of Neurosurgery, Iwate Prefectural Chubu Hospital, Kitakami, Iwate, Japan
}

\section{ABSTRACT}

Although posterior reversible encephalopathy syndrome (PRES) is rarely associated with subarachnoid hemorrhage, to our knowledge, rupture of a concomitant cerebral aneurysm following PRES has not been reported. We describe a patient with atypical PRES involving the brainstem, thalamus, and periventricular white matter without cortical or subcortical edema of the parietooccipital lobe on magnetic resonance imaging, with rupture of a concomitant cerebral aneurysm. Preexisting extremely high blood pressure may trigger atypical PRES, and failure to lower blood pressure may lead to a concomitant aneurysm rupture. In the future treatment of hypertensive urgency with a recurrence of symptoms and mean arterial blood pressure $>150 \mathrm{mmHg}$, it is advisable to immediately hospitalize the patient for aggressive blood pressure management, especially if PRES is suspected based on clinical and radiological features.

Key words: Acute hypertension, aneurysm, posterior reversible encephalopathy syndrome, subarachnoid hemorrhage

\section{Introduction}

Posterior reversible encephalopathy syndrome (PRES) is a clinico-radiological entity associated with various conditions such as eclampsia, severe hypertension, and cytotoxic medications. Clinically, the syndrome is usually reversible, ${ }^{[1]}$ but delayed diagnosis and failure to treat hypertension promptly may lead to ischemia, hemorrhage, and nonaneurysmal subarachnoid hemorrhage (SAH) resulting in permanent damage to the affected brain tissue ${ }^{[2]}$ Radiologically, PRES typically affects the cortex and subcortical white matter within the parietooccipital or posterior frontal lobes. The brainstem, thalamus, basal ganglia, and periventricular white matter are less commonly affected. ${ }^{[3-7]}$

\section{Address for correspondence:}

Dr. Hiroshi Kashimura, Department of Neurosurgery, Iwate

Prefectural Chubu Hospital, 17-10 Murasakino, Kitakami, Iwate 024-8507, Japan.

E-mail: h-kashimura@pref.iwate.jp

\begin{tabular}{|l|l|}
\hline \multicolumn{2}{|c|}{ Access this article online } \\
\hline Quick Response Code: & Website: \\
\hline & www.ruralneuropractice.com \\
\hline & \\
\hline & \\
\hline
\end{tabular}

We describe a patient with atypical PRES involving the brainstem, thalamus, and periventricular white matter without cortical or subcortical edema of the parietooccipital lobe on magnetic resonance (MR) imaging, with rupture of a concomitant cerebral aneurysm.

\section{Case Report}

A 47-year-old woman with a 24-day history of hypertension followed by a headache was referred to our neurosurgery department. Three days prior, she had been started on oral antihypertensive therapy with azilsartan $20 \mathrm{mg}$ a day at a local hospital, and the patient's headache improved gradually with the antihypertensive therapy. On arrival, her blood pressure was 197/106 mmHg. No focal neurological abnormality was noted. Fluid-attenuated inversion recovery (FLAIR) and T2-weighted MR imaging revealed high-intensity

This is an open access article distributed under the terms of the Creative Commons Attribution-NonCommercial-ShareAlike 3.0 License, which allows others to remix, tweak, and build upon the work non-commercially, as long as the author is credited and the new creations are licensed under the identical terms.

For reprints contact: reprints@medknow.com

How to cite this article: Nanba T, Kashimura H, Saura H, Takeda M. Subarachnoid hemorrhage due to ruptured intracranial aneurysm following posterior reversible encephalopathy syndrome. J Neurosci Rural Pract 2016;7:440-2. 
lesions in the pons, bilateral thalamus, bilateral basal ganglia, and periventricular white matter. There was no evidence of SAH. Diffusion-weighted MR imaging detected no signal abnormalities in these regions, except in the bilateral basal ganglia, which exhibited low intensity [Figure 1]. MR angiography revealed a saccular aneurysm located in the right internal carotid artery [Figure 2]. Arterial vasospasm was not observed in the anterior or posterior circulation. She was not admitted to our hospital at this time because MR imaging did not reveal SAH or acute ischemic lesions, and neurological examination revealed no deficits. Two days later, she experienced severe headache and was admitted to our hospital. On arrival, her blood pressure was $274 / 133 \mathrm{mmHg}$. She was subsequently started on intravenous nicardipine. Her arterial blood pressure was maintained between 115/63 and $141 / 76 \mathrm{mmHg}$. The patient was alert and fully oriented without focal neurological deficits. Initial computed tomography failed to diagnose SAH. FLAIR revealed slightly high-density lesions in the right side of the sylvian cistern and basal cistern consistent with SAH as well as high-intensity lesions in the pons, bilateral thalamus, bilateral basal ganglia, and periventricular white matter [Figure 3]. Two days after the onset of $\mathrm{SAH}$, she successfully underwent neck clipping of an aneurysm via a craniotomy under general anesthesia. Intraoperatively, we did not identify local hemosiderin staining of the pial surface immediately adjacent to the

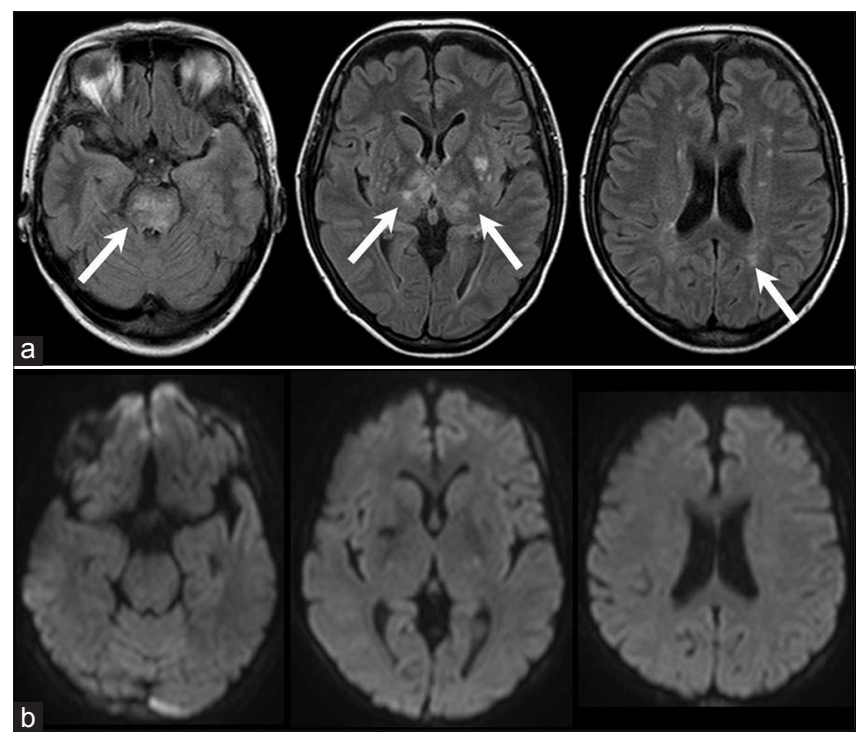

Figure 1: Fluid-attenuated inversion recovery magnetic resonance images showing slightly high-density lesions in the right side of the sylvian cistern and the basal cistern consistent with subarachnoid hemorrhage (white arrows) and high-intensity lesions in the pons, bilateral thalamus, bilateral basal ganglia, and periventricular white matter (a); diffusion-weighted magnetic resonance images showing no signal abnormalities in the pons, bilateral thalamus, or periventricular white matter, but low intensity in the bilateral basal ganglia (b) aneurysm dome suggestive of a previous aneurysm rupture. Postoperatively, the patient experienced no cerebral ischemic events. Her systolic and diastolic blood pressure were controlled with intravenous nicardipine and/or oral antihypertensive agents to $<150$ and $100 \mathrm{mmHg}$, respectively. Follow-up MR imaging performed 2 weeks later revealed diminution of the lesions [Figure 4].

\section{Discussion}

In the present case, FLAIR MR imaging revealed high-intensity lesions in the pons, bilateral thalamus, and periventricular white matter, although diffusion-weighted MR imaging revealed no abnormal findings in those lesions. MR imaging repeated 2 weeks later revealed diminution of the lesions. These radiological findings may be attributed to atypical PRES. Clinically, the patient's headache improved gradually with antihypertensive therapy. These findings were also consistent with PRES.

PRES typically affects the cortex and subcortical white matter within the parietooccipital or posterior frontal lobes. The brainstem, thalamus, and basal ganglia are less commonly affected..$^{[3-7]}$ The exact mechanisms underlying selective vulnerability of the brainstem, basal ganglia, and thalamus are not understood. It has been reported that mild hypertension induces edema predominantly in the supratentorial white matter, whereas severe hypertension induces vasogenic edema in the infratentorial structures, basal ganglia, and thalamus, and the mean arterial blood pressure of patients with severe hypertension typically exceeds $150 \mathrm{mmHg}$, which suggests that severe acceleration of hypertension is required for atypical PRES. ${ }^{[5]}$ In

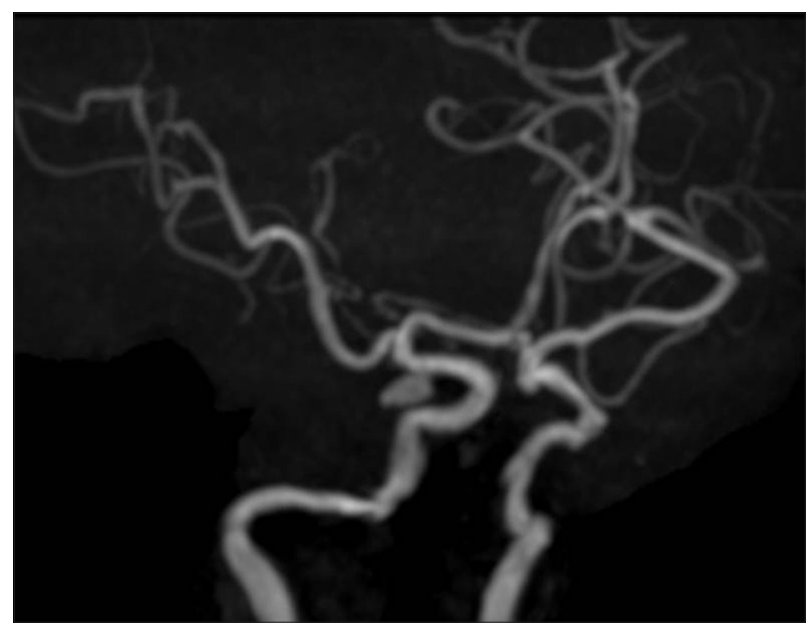

Figure 2: Magnetic resonance angiography showing a saccular aneurysm located in the right internal carotid artery 


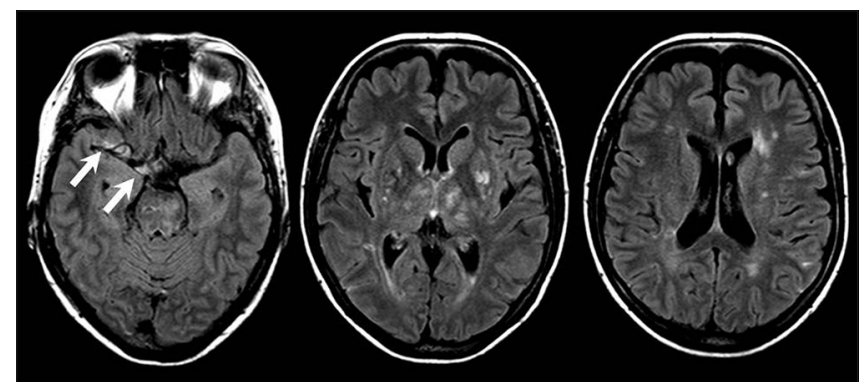

Figure 3: Fluid-attenuated inversion recovery magnetic resonance images showing a high-intensity lesion in the right sylvian cistern and the right side of the basal cistern consistent with subarachnoid hemorrhage (white arrows)

this case, the patient's mean arterial pressure blood pressure exceeded $150 \mathrm{mmHg}$ before and after oral antihypertensive therapy. Preexisting extremely high blood pressure may have triggered atypical PRES.

There have previously been several reports of PRES following SAH. ${ }^{[8-10]}$ This syndrome is related to hypervolemia, hypertension, and hemodilution therapy (triple $\mathrm{H}$ ) for cerebral vasospasm following SAH. Cerebral vasospasm is a complication of SAH. Triple $\mathrm{H}$ therapy has been used to treat and reduce the risk of vasospasm. Kuroda et al. reported a case involving a patient who developed PRES, prior to induction of hypertensive therapy, 2 days after the onset of an SAH due to a ruptured intracranial aneurysm. ${ }^{[8]}$ Acute hypertension caused by a catecholamine surge at the time of aneurysm rupture stimulates a sympathetic storm, leading to an abrupt increase in blood pressure. This acute hypertension may have resulted in PRES after SAH. In this case, initial MR images showed atypical PRES without SAH. Furthermore, we did not intraoperatively identify local hemosiderin staining of the pial surface immediately adjacent to the aneurysm dome suggestive of a previous aneurysm rupture. Hypertension is one of the risk factors for SAH. Thus, we speculated that sustained high blood pressure after onset of atypical PRES can lead to concomitant aneurysm rupture. Although PRES is rarely associated with SAH, to our knowledge, rupture of a concomitant cerebral aneurysm following PRES has not been reported.

\section{Conclusion}

We describe a patient with atypical PRES with rupture of a concomitant cerebral aneurysm in the early stages of antihypertensive therapy for PRES involving the brainstem, thalamus, and periventricular white matter without cortical or subcortical edema of the parietooccipital lobe on MR imaging. Although the syndrome is generally a reversible phenomenon with

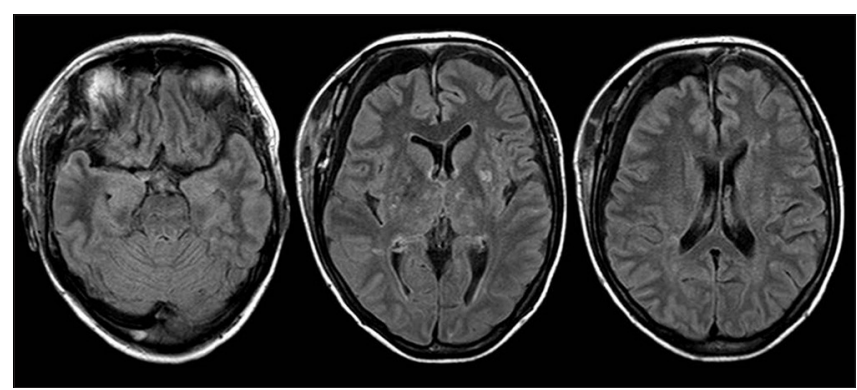

Figure 4: Follow-up magnetic resonance images performed 2 weeks later showing diminution of the lesions

antihypertensive therapy, failure to lower blood pressure and delayed diagnosis may have led to the concomitant aneurysm rupture in this patient. In the future treatment of hypertensive urgency with recurrence of symptoms and mean arterial blood pressure $<150 \mathrm{mmHg}$, it is advisable to immediately hospitalize the patient for aggressive blood pressure management, especially if PRES is suspected based on clinical and radiological features.

\section{Financial support and sponsorship Nil.}

\section{Conflicts of interest}

There are no conflicts of interest.

\section{References}

1. Stott VL, Hurrell MA, Anderson TJ. Reversible posterior leukoencephalopathy syndrome: A misnomer reviewed. Intern Med J 2005;35:83-90.

2. Hefzy HM, Bartynski WS, Boardman JF, Lacomis D. Hemorrhage in posterior reversible encephalopathy syndrome: Imaging and clinical features. AJNR Am J Neuroradiol 2009;30:1371-9.

3. Karakis I, Macdonald JA, Stefanidou M, Kase CS. Clinical and radiological features of brainstem variant of hypertensive encephalopathy. J Vasc Interv Neurol 2009;2:172-6.

4. Kitaguchi H, Tomimoto H, Miki Y, Yamamoto A, Terada K, Satoi H, et al. A brainstem variant of reversible posterior leukoencephalopathy syndrome. Neuroradiology 2005;47:652-6.

5. Kumai Y, Toyoda K, Fujii K, Ibayashi S. Hypertensive encephalopathy extending into the whole brainstem and deep structures. Hypertens Res 2002;25:797-800.

6. McKinney AM, Jagadeesan BD, Truwit CL. Central-variant posterior reversible encephalopathy syndrome: Brainstem or basal ganglia involvement lacking cortical or subcortical cerebral edema. AJR Am J Roentgenol 2013;201:631-8.

7. Shimizu Y, Tha KK, Iguchi A, Cho Y, Yoshida A, Fujima N, et al. Isolated posterior fossa involvement in posterior reversible encephalopathy syndrome. Neuroradiol J 2013;26:514-9.

8. Kuroda H, Kashimura H, Murakami T, Endo H, Mase T, Ogasawara K. Early onset of PRES in a patient with a subarachnoid haemorrhage due to a ruptured intracranial aneurysm. Br J Neurosurg 2014;28:785-6.

9. Amin-Hanjani S, Schwartz RB, Sathi S, Stieg PE. Hypertensive encephalopathy as a complication of hyperdynamic therapy for vasospasm: Report of two cases. Neurosurgery 1999;44:1113-6.

10. Jang HW, Lee HJ. Posterior reversible leukoencephalopathy due to "triple H” therapy. J Clin Neurosci 2010;17:1059-61. 